\title{
Impacts of the SOAT1 genetic variants and protein expression on HBV-related hepatocellular carcinoma
}

\author{
Yulong Chen ${ }^{1 \dagger}$, Xunjun Yang ${ }^{1,2+}$, Yao Chen ${ }^{3}$, Guorong Chen ${ }^{3}$, Cheryl A. Winkler ${ }^{4}$, Ping An ${ }^{4^{*}}$ (D) and Jianxin Lyu ${ }^{1,5^{*}}$
}

\begin{abstract}
Background: Hepatitis B virus (HBV)-related hepatocellular carcinoma (HCC) remains a major public health problem and its pathogenesis remains unresolved. A recent proteomics study discovered a lipid enzyme Sterol Oacyltransferase (SOAT1) involvement in the progression of HCC. We aimed to explore the association between SOAT1 genetic variation and HCC.

Methods: We genotyped three exonic SOAT1 variants (rs10753191, V323V; rs3753526, L475L; rs13306731, Q526R) tagging most variations in the gene, in 221 HCC patients and 229 healthy individuals, to assess the impact of SOAT1 gene variation on risk of HCC occurrence. We further conducted immunohistochemistry to compare SOAT1 protein expression levels in 42 paired tumor and adjacent non-tumor tissues.

Results: We found that rs 10753191 (Odds ratio $(O R)=0.58, P=0.04$ ) and a haplotype TGA (OR=0.40, $P=0.01$ ) were associated with reduced HCC risk after adjusting for lipid levels. In the immunohistochemistry experiment, we found that the protein expression of SOAT1 was significantly increased in the tumor compared with adjacent tissue $(P<0.001)$.

Conclusion: This study revealed for the first time SOAT1 genetic variation that associates with host susceptibility to HCC occurrence. Our results suggest a role of SOAT1 in the HCC development, which warrants further elucidation.
\end{abstract}

Keywords: Hepatocellular carcinoma, SOAT1, Single nucleotide polymorphism, Susceptibility

\section{Background}

Liver cancer is the second major cause of mortality for all types of cancer worldwide. Hepatocellular carcinoma (HCC) represents the largest proportion in liver cancer [1]. HCC incidence rates vary globally, with the majority of HCC cases occurring in East Asia and sub-Saharan Africa, due to the high prevalence of Hepatitis B virus (HBV) and hepatitis $\mathrm{C}$ virus $(\mathrm{HCV})$. The United States

\footnotetext{
* Correspondence: ping.an@nih.gov; jxlu313@163.com

†Yulong Chen and Xunjun Yang contributed equally to this work.

${ }^{4}$ Basic Science Program, Frederick National Laboratory for Cancer Research, Frederick, MD, USA

'Key Laboratory of Laboratory Medicine, Ministry of Education, Zhejiang Provincial Key Laboratory of Medical Genetics, Wenzhou Medical University, Wenzhou, Zhejiang, China

Full list of author information is available at the end of the article
}

and Northern Europe have a low HCC incidence but it has been increasing in recent years [2]. The major risk factors of HCC include chronic HBV and HCV infection, aflatoxin exposure, alcohol abuse, and non-alcoholic fatty liver disease (NAFLD). Among these factors, higher NAFL $\mathrm{D}$ prevalence is considered one of the key factors related to the increasing incidence of $\mathrm{HCC}$ in the low incidence areas and is expected to become the major cause of HCC in the future $[3,4]$.

Sterol O-acyltransferase (SOAT), also known as acylCoA: cholesterol acyltransferase (ACAT), is located in the endoplasmic reticulum membrane where it catalyzes cholesterol into cholesterol esters and plays an essential role in cholesterol homeostasis and bile acid biosynthesis $[5,6]$. SOAT-mediated esterification of cholesterol prevents

(c) The Author(s). 2021 Open Access This article is licensed under a Creative Commons Attribution 4.0 International License, which permits use, sharing, adaptation, distribution and reproduction in any medium or format, as long as you give appropriate credit to the original author(s) and the source, provide a link to the Creative Commons licence, and indicate if changes were made. The images or other third party material in this article are included in the article's Creative Commons licence, unless indicated otherwise in a credit line to the material. If material is not included in the article's Creative Commons licence and your intended use is not permitted by statutory regulation or exceeds the permitted use, you will need to obtain permission directly from the copyright holder. To view a copy of this licence, visit http://creativecommons.org/licenses/by/4.0/. The Creative Commons Public Domain Dedication waiver (http://creativecommons.org/publicdomain/zero/1.0/) applies to the data made available in this article, unless otherwise stated in a credit line to the data. 
the toxic accumulation of free cholesterol in cell membrane [7]. SOAT1 is ubiquitously expressed in all tissues except the intestine. SOAT1 is the major enzyme with higher expression level and plays an important role in cholesterol homeostasis [5, 8-10]. A recent proteomic study performed in early-stage HBV-HCC patients revealed that SOAT1 plays an important role in a severe subtype of HCC [11]. They reported that HCC patients with more aggressive tumors and poorer prognosis had disrupted cholesterol metabolism and higher SOAT1 expression [11].

SOAT1 in HCC has been considered as a new promising target for HCC diagnosis and treatment $[12,13]$. SOAT1 protein expression in HCC cell lines and inhibition of patient-derived tumor xenograft models demonstrated that SOAT1 suppression may be an effective HCC treatment [11]. Single nucleotide polymorphisms (SNPs) of SOAT1 have been associated with cholesterol metabolism [14, 15]. However, the association between SOAT1 SNPs and HCC has not been explored. Therefore, to assess whether SOAT1 is related to risk of HCC occurrence, we explored the association of SOAT1 gene missense variants with HCC susceptibility in a casecontrol design of biopsy proven HCC patients and healthy controls. To our knowledge, this is the first study to report a relationship between SOAT1 genetic variants and $\mathrm{HCC}$.

\section{Methods}

\section{Study subject}

The study included 221 cases diagnosed with HCC and 229 healthy control individuals from First Affiliated Hospital of Wenzhou Medical University between January 2010 and March 2019. There were 160 HBV infected HCC patients (72.4\%) among all HCC cases. The selfreported ethnicity of participants was Han Chinese. All cases were confirmed by histopathology to have HCC. Inclusion criteria for healthy controls was no evidence of current hepatitis virus infection, no history of liver or other metabolic diseases, and no other malignancies. We obtained demographic and clinical data from review of medical charts.

The study was conducted in accordance with the Declaration of Helsinki. The Ethics Committee of Wenzhou Medical University approved this study. Informed consents were obtained from individuals of healthy controls. An IRB exemption was obtained from the National Institutes of Health Office of Human Subjects Research (OHSRP Review \#12836) for using archived pathological specimens and the de-identified health information.

\section{Samples}

We obtained all samples from the First Affiliated Hospital of Wenzhou Medical University. Achieved formalin-fixed and paraffin-embedded (FFPE) tissue from HCC patients were obtained from the Pathology Department and DNA was extracted from using the phenol extract method [16, 17]. Tumor grading and staging were classified by Barcelona.

Clinic liver Cancer Staging system (BCLC) [18]. We used the Universal Genomic DNA Extraction Kit Ver3.0 (Takara Bio, Japan) to extract genomic DNA from peripheral whole blood of healthy individuals.

\section{SNP selection}

We selected variant sites from NCBI dbSNP and 1000Genomes database, based on the following criteria: (i) haplotype tagging SNPs; (ii) SNPs in the exonic regions of SOAT1; (iii) minor allele frequency (MAF) > 0.02 in Han Chinese from Beijing (CHB) in the 1000Genomes project database. Data from 1000Genomes indicated that most common variants in SOAT1 were in strong linkage disequilibrium in three typical populations from China, Europe and Africa (Fig. S1). Then, we selected rs10753191 (synonymous amino acid change V323V), rs3753526 (synonymous amino acid change L475L) and rs13306731(nonsynonymous change Q526R (Gln526Arg)) from the $65 \mathrm{~kb} S O A T 1$ gene, which covers a haplotype block approximately $7.8 \mathrm{~kb}$ from exon10 to exon16 (Fig. 1, Table 2). We analyzed the linkage disequilibrium of all 385 SOAT1 variants in the CHB population available in the 1000 Genomes project database. These SNPs are in strong linkage disequilibrium (Average $\mathrm{D}^{\prime}: 0.934 \pm 0.156$ (mean $\pm \mathrm{sd}$ ), Fig. 1). We included all exonic SNPs in the SOAT1 gene with MAF $>0.02$ in CHB except one SNP rs11576517 (P199P) which was in high LD with $\mathrm{rs} 10753191\left(\mathrm{D}^{\prime}=0.84, r^{2}=0.42\right)$. rs7547733 (F258F), which is common in the European population $(\mathrm{MAF}=$ 0.20 ) is absent in east Asians (including CHB).

\section{Genotyping}

We conducted SNPs genotyping with TaqMan SNP Genotyping Assays using a real-time quantitative PCR method on a StepOnePlus Real-Time PCR System (Applied Biosystems, Foster City, California, USA) following Applied Biosystems protocols. Genotype call were made using the TaqMan Genotyper Software (Applied Biosystems). All the reactions were carried out in a total volume of 10ul containing TaqPath ProAmp Master Mix, SNP genotyping assay (20x), DNA-free water and genomic DNA. The PCR parameters were set as follows: $60^{\circ} \mathrm{C}$ for $60 \mathrm{~s} ; 95^{\circ} \mathrm{C}$ for 30s; 40 cycles at $95^{\circ} \mathrm{C}$ for $15 \mathrm{~s}$ and $60^{\circ} \mathrm{C}$ for $60 \mathrm{~s} ; 60^{\circ} \mathrm{C}$ for $30 \mathrm{~s}$. About $10 \%$ random samples were duplicated for genotyping and the results were $100 \%$ concordant.

\section{Immunohistochemistry (IHC) for SOAT1 protein expression}

We processed FFPE tissue samples to $3.5 \mu \mathrm{m}$ to detect the expression of SOAT1 by IHC. Then we 


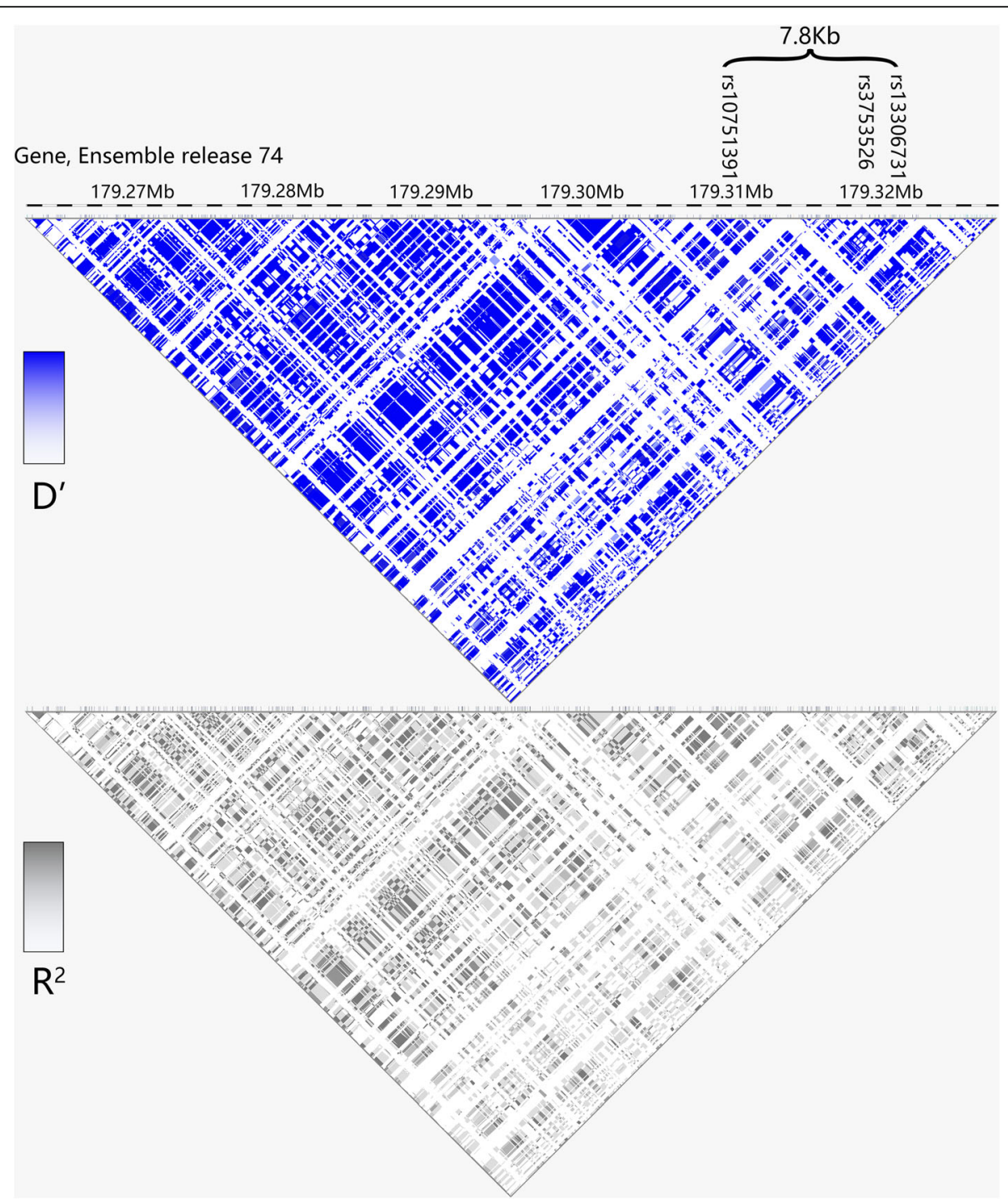

Fig. 1 Linkage disequilibrium of SNPS in the SOAT1 gene and locations of three SNPs tested in this study. The total length of the SOAT1 gene is $65 \mathrm{~Kb}$, with three SNPs in this study covering a 7.8Kb region. The figure shows the degree of linkage $\left(D^{\prime}\right)$ and correlation $\left(r^{2}\right)$ of SNPs in the gene region of CHB. Most SNPS of SOAT1 gene are in strong linkage disequilibrium (D': $0.934 \pm 0.156)$

deparaffinized, rehydrated, and exposed those sections to $3 \% \mathrm{H}_{2} \mathrm{O}_{2}$ solution for $10 \mathrm{~min}$ and performed antigen retrieval in the citrate buffer, followed by incubation with anti-SOAT1 (ABN66, Sigma) at 1:500 dilution at $4{ }^{\circ} \mathrm{C}$ overnight. Anti-rabbit secondary antibody was then applied to sections for $25 \mathrm{~min}$ at $37^{\circ} \mathrm{C}$. After that we conducted visualization with Diaminobenzidine (ZSGB-Bio). The intensity of IHC results was defined as negative (0), low (1), medium (2) and high (3), while the percentage of stained cells were defined as none (0), $1-25 \%$ (1), $25-50 \%$ (2) $50-75 \%$ (3) and $>75 \%$ (4). According to the product of intensity and percentage, we classified samples as low expression (0-6) and high expression (8-12).

\section{Statistical analysis}

All statistical analyses were performed with $\mathrm{R}$ language [20] using RStudio Version 1.2.1335. We performed linkage disequilibrium (LD) and haplotype analysis with LD heatmap package [21] and Haplo.stats package [22]. The genotype distribution of all SNPs among control samples were conformed to Hardy-Weinberg equilibrium. Baseline characteristics of study subjects was d escribed as mean \pm standard deviation (SD) or percentages. Significance of different groups was calculated with Fisher's exact test or logistic regression. We conducted log transformation to non-normal distribution data before Wilcoxon rank sum test and logistic regression. Finally, we applied Fisher's exact test and Wilcoxon-signed rank sum test to IHC 
scores. Results were considered significant for $P$ value less than 0.05 and all tests were two-tailed.

\section{HCC data from TCGA}

Accessible transcriptomic data of $364 \mathrm{HCC}$ patients with overall survival (OS) data from the TCGA were analyzed. Differences in overall survival (OS) were tested by Cox proportional hazards regression for the high or low, as divided by median, of SOAT1 mRNA levels measured by RNA-seq. KaplanMeier survival plots with hazard rates (HR) and log-rank $p$ values were calculated and plotted, separately for the White and Asian ethnic groups and also for the all ethnic groups (Plus black, $n=17$ ), as implemented in K-M plotter [23].

\section{Results}

\section{Characteristic of study subjects}

The characteristics of the HCC cases $(n=221)$ and healthy controls $(n=229)$ are presented in Table 1. Age, sex or BMI distribution were similar between cases and controls $(P>0.05$, Table 1$)$. In the lipid profiles comparison, we observed that low density lipoprotein (LDL), high density lipoprotein (HDL), total cholesterol (TC) and triglyceride (TG) were all lower in HCC cases than healthy controls $(P<0.001$, Table 1$)$.

\section{Association of SOAT1 SNPs with the risk of HCC}

The primary information of three SOAT1 SNPs (rs10753191, rs3753526, rs13306731) is displayed in Table 2. MAFs of SNPs in our controls were similar to MAFs in Han Chinese individuals (CHB population) from the 1000Genomes project [19].

The association of SNPs with HCC risk was presented in Table 3. Genotyping results showed rs10753191 and rs3753526 are in near absolute positive LD and therefore rs10753191 is a proxy for rs3753526. We evaluated the associations of the SNPs with HCC status using dominant, recessive, additive genetic models. We observed no significant associations in the minimally adjusted model adjusting for age and sex; however, after adjusting for

Table 1 Characteristics of HCC cases and controls

\begin{tabular}{|c|c|c|c|}
\hline & Case $(n=221)$ & Control $(n=229)$ & $P$ value \\
\hline Age, years ${ }^{a}$ & $57.29 \pm 10.88$ & $55.59 \pm 11.64$ & 0.109 \\
\hline $\operatorname{Sex}^{\mathrm{b}}$ & $181(81.90 \%)$ & 170 (74.24\%) & 0.065 \\
\hline $\mathrm{BMI}^{\mathrm{a}}$ & $23.04 \pm 2.97$ & $23.07 \pm 2.25$ & 0.936 \\
\hline $\mathrm{TC}(\mathrm{mmol} / \mathrm{dl})^{\mathrm{a}}$ & $3.82 \pm 1.20$ & $4.92 \pm 0.85$ & $<0.001$ \\
\hline $\mathrm{TG}(\mathrm{mmol} / \mathrm{dl})^{\mathrm{a}}$ & $1.12 \pm 0.77$ & $1.44 \pm 0.85$ & $<0.001$ \\
\hline $\mathrm{LDL}(\mathrm{mmol} / \mathrm{dl})^{\mathrm{a}}$ & $2.32 \pm 0.90$ & $2.77 \pm 0.73$ & $<0.001$ \\
\hline $\mathrm{HDL}(\mathrm{mmol} / \mathrm{dl})^{\mathrm{a}}$ & $0.97 \pm 0.36$ & $1.43 \pm 0.36$ & $<0.001$ \\
\hline
\end{tabular}

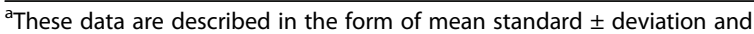
compared with t test

bercentage and $\mathrm{Chi}^{2}$ test lipid levels, carriers of rs10753191 $\mathrm{T}$ had a lower risk for HCC $(\mathrm{OR}=0.583$, 95\% Confidence Interval (CI) 0.348 $0.977, P=0.041$, dominant model).

\section{Association of SOAT1 haplotype consisting of three SNPs with the risk of $\mathrm{HCC}$}

All SNP data of patients and healthy controls was combined to determine the extent of linkage disequilibrium for the three SNPs (Additional file 1 Table S1). All three SNPs were in strong linkage disequilibrium $\left(\mathrm{D}^{\prime}=1, r^{2}>\right.$ 0.7). Additionally, we validated this result with other three typical populations from 1000 Genomes [19] (CHB, CEU and YRI) and all three SNPs were assigned to the same haplotype block (Additional file $1 \mathrm{Fig} . \mathrm{S} 1$ ).

Next, we used Haplo.stats [22] to obtain the haplotype assignments of samples. The association of haplotypes with HCC risk is presented in Table 4. None of the three haplotypes was associated with HCC susceptibility in the minimally adjusted model, but after adding lipids to the model, haplotype TGA was associated with decreased risk of $\mathrm{HCC}(\mathrm{OR}=0.395,95 \% \mathrm{CI}=0.191-0.817, \quad P=$ 0.012) (Table 4).

\section{Association of SOAT1 SNPs and haplotype with HCC characteristics (Table 5)}

We compared characteristics of HCC patients (including LDL, HDL, TC, TG, tumor size, alpha-fetoprotein level (AFP), HBV status and pathological stage) between different SOAT1 genotypes and haplotypes. There were no significant differences in lipid levels, triglycerides, tumor size, stage, or HBV status by genotype or haplotype. Carriers of the variant alleles for rs10753191 and rs13306731 were more likely to have elevated AFP levels ( $P=0.014$ and $P=0.010$, respectively). Moreover, haplotype TGG was associated with a tendency of lower AFP level in the minimally adjusted model $(P=0.042)$, but when lipids were added to the model the association was attenuated $(P=0.065$, Table 5$)$. We did not find significant association of the SOAT1 SNPs and haplotypes with lipid levels in HCC patients or healthy controls $(P>0.05$, Additional file 1 Table S2).

\section{Association of SNPs with SOAT1 protein expression in HCC tumor and liver tissue}

We measured the SOAT1 protein expression by immunohistochemistry (IHC) in HCC liver tissue samples and paired non-tumor tissue samples from 42 patients. Representative staining results are shown in Fig. 2. Immunoreactivity were mainly seen on the membrane plasma of tumor cells. A small number of lymphocytes were also weakly stained. We compared the differences in expression between hepatocytes and hepatocarcinoma cells. We conducted paired Wilcoxon signed-rank test for IHC score differences and found SOAT1 has a 
Table 2 Characteristics of SOAT1 SNPS and haplotypes

\begin{tabular}{|c|c|c|c|c|}
\hline SNP & rs10753191 & rs3753526 & rs13306731 & \\
\hline Position $^{a}$ & $179,312,752$ & $179,319,541$ & $179,320,578$ & \\
\hline REF & C & C & A & \\
\hline ALT & $\mathrm{T}$ & G & G & \\
\hline Amino acid change & V323V & L475L & Q526R & \\
\hline TF binding ${ }^{b}$ & RFX5 & No & No & \\
\hline$M A F^{c}$ in $C H B^{d}$ & 0.403 & 0.408 & 0.306 & \\
\hline MAF in $C E U^{d}$ & 0.091 & 0.040 & 0.040 & \\
\hline MAF in YRI ${ }^{d}$ & 0.361 & 0.116 & 0 & \\
\hline MAF in the control group & 0.394 & 0.394 & 0.316 & \\
\hline Hardy-Weinberg Equilibrium $P$ value in the control group & 0.197 & 0.197 & 0.790 & \\
\hline Haplotype & This Study & $\mathrm{CHB}$ & CEU & YRI \\
\hline CCA & 0.628 & 0.592 & 0.909 & 0.639 \\
\hline TGG & 0.303 & 0.306 & 0.040 & 0.000 \\
\hline TGA & 0.069 & 0.097 & 0.000 & 0.116 \\
\hline CGA & 0.000 & 0.005 & 0.000 & 0.000 \\
\hline TCA & 0.000 & 0.000 & 0.051 & 0.245 \\
\hline
\end{tabular}

${ }^{a_{N}}$ C 000001.10 (chr1), Feb 2009 GRCh37/hg19)

${ }^{\mathrm{b}}$ Transcriptional factor (TF) binding

'MAF: Minor allele frequency

${ }^{\mathrm{d}}$ Genotype data of CHB, CEU, YRI are all come from 1000Genomes [19]

CHB individuals from unrelated Han Chinese individuals from Beijing, China, CEU residents of Utah with western European ancestry, YRI individuals from Yoruba population of African origin

Table 3 Associations of SOAT1 SNPS with risk of HBV-HCC

\begin{tabular}{|c|c|c|c|c|c|c|c|c|}
\hline & $\begin{array}{l}\text { Case } \\
(n=221)\end{array}$ & $\begin{array}{l}\text { Control } \\
(n=229) \\
\end{array}$ & $P$ value & $\mathrm{OR}(95 \% \mathrm{Cl})$ & $P$ value $^{\dagger}$ & $\mathrm{OR}(95 \% \mathrm{Cl})^{\dagger}$ & $P$ value $^{\ddagger}$ & $\mathrm{OR}(95 \% \mathrm{Cl})^{\ddagger}$ \\
\hline \multicolumn{9}{|l|}{ rs10753191 } \\
\hline $\mathrm{CC}$ & $94(42.5 \%)$ & 81 (35.4\%) & Ref. & / & Ref. & / & Ref. & / \\
\hline $\mathrm{CT}$ & 97 (43.9\%) & $118(51.5 \%)$ & 0.103 & $0.708(0.475-1.057)$ & 0.107 & $0.716(0.477-1.075)$ & 0.035 & $0.564(0.331-0.960)$ \\
\hline$\pi$ & $30(13.6 \%)$ & 30 (13.1\%) & 0.655 & $0.862(0.479-1.550)$ & 0.547 & $0.833(0.460-1.508)$ & 0.483 & $0.747(0.331-1.689)$ \\
\hline Dominant & / & / & 0.123 & $0.739(0.506-1.081)$ & 0.123 & $0.739(0.504-1.085)$ & 0.040 & $0.583(0.508-2.177)$ \\
\hline Recessive & / & / & 0.891 & $1.042(0.605-1.794)$ & 0.965 & $1.012(0.586-1.749)$ & 0.893 & $1.051(0.508-2.177)$ \\
\hline Additive & / & / & 0.237 & $0.840(0.634-1.122)$ & 0.277 & $0.858(0.651-1.131)$ & 0.156 & $0.764(0.526-1.108)$ \\
\hline \multicolumn{9}{|l|}{ rs13306731 } \\
\hline AA & $107(48.4 \%)$ & $106(46.3 \%)$ & Ref. & / & Ref. & / & Ref. & / \\
\hline$A G$ & 95 (43.0\%) & $106(46.3 \%)$ & 0.557 & $0.888(0.604-1.306)$ & 0.626 & $0.908(0.614-1.341)$ & 0.496 & $0.837(0.502-1.397)$ \\
\hline GG & $19(8.6 \%)$ & 17 (7.4\%) & 0.858 & $1.107(0.546-2.246)$ & 0.865 & $1.064(0.519-2.185)$ & 0.687 & $1.207(0.483-3.020)$ \\
\hline Dominant & / & / & 0.706 & $0.918(0.634-1.330)$ & 0.714 & $0.932(0.642-1.355)$ & 0.613 & $0.881(0.538-1.442)$ \\
\hline Recessive & / & / & 0.729 & $1.173(0.593-2.320)$ & 0.706 & $1.141(0.574-2.268)$ & 0.558 & $1.299(0.541-3.122)$ \\
\hline Additive & / & / & 0.750 & $0.963(0.513-1.563)$ & 0.898 & $0.981(0.731-1.317)$ & 0.892 & $0.974(0.663-1.430)$ \\
\hline
\end{tabular}

${ }^{\dagger}$ Adjusted by age, gender

${ }^{\ddagger}$ Adjusted by age, gender, HDL, LDL, TC and TG

Dominant model: CC vs. CT + TT for rs10753191; AA vs. AG + GG for rs13306731

Recessive model: $\Pi T$ vs. $C C+C T$ for rs10753191; GG vs. AA+AG for rs 13306731

Additive model: CC vs. CT vs. TT for rs10753191; GG vs. AA vs. AG vs. GG for rs13306731 
Table 4 Associations of the SOAT1 haplotypes with the risk of HCC

\begin{tabular}{lllllllll}
\hline & Case & Control & $\boldsymbol{P}$ value & OR(95\% Cl) & $\boldsymbol{P}_{\text {value }}^{\dagger}$ & OR(95\% Cl ${ }^{\dagger}$ & $\boldsymbol{P ~ v a l u e ~}^{\ddagger}$ & OR(95\% Cl$^{\ddagger}$ \\
\hline CCA & 285 & 280 & Ref. & $/$ & Ref. & $/$ & Ref. & $/$ \\
TGA & 24 & 38 & 0.083 & $0.620(0.363-1.062)$ & 0.056 & $0.587(0.340-1.013)$ & $\mathbf{0 . 0 1 2}$ & $0.395(0.191-0.817)$ \\
TGG & 133 & 140 & 0.659 & $0.933(0.699-1.246)$ & 0.647 & $0.934(0.698-1.250)$ & 0.585 & $0.900(0.618-1.312)$ \\
\hline
\end{tabular}

${ }^{\dagger}$ Model 1, adjusted for age and sex

${ }^{\ddagger}$ Model 2, model 1 with additional adjustment of HDL, LDL, TC and TG

Table 5 Association of the SOAT1 SNPs with the characteristic of HCC patients, dominant model

\begin{tabular}{|c|c|c|c|c|c|c|c|}
\hline & \multicolumn{2}{|c|}{ Reference genotype } & \multicolumn{2}{|c|}{ Variant genotypes } & \multicolumn{2}{|l|}{$P$ value $^{\dagger}$} & $P$ value $^{\ddagger}$ \\
\hline $\begin{array}{l}\text { rs10753191/ } \\
\text { rs3753526 }\end{array}$ & \multicolumn{2}{|l|}{$\begin{array}{l}\mathrm{CC} / \mathrm{CC} \\
(n=94)\end{array}$} & \multicolumn{2}{|l|}{$\begin{array}{l}\text { CT\&TT/CG\&GG } \\
(n=127)\end{array}$} & & & \\
\hline LDL (mmol/dl) & \multicolumn{2}{|l|}{$2.276 \pm 0.879$} & \multicolumn{2}{|l|}{$2.353 \pm 0.911$} & \multicolumn{2}{|l|}{0.608} & 0.606 \\
\hline $\mathrm{HDL}(\mathrm{mmol} / \mathrm{dl})$ & \multicolumn{2}{|l|}{$0.945 \pm 0.391$} & \multicolumn{2}{|l|}{$0.984 \pm 0.328$} & \multicolumn{2}{|l|}{0.248} & 0.237 \\
\hline $\mathrm{TC}(\mathrm{mmol} / \mathrm{dl})$ & \multicolumn{2}{|l|}{$3.803 \pm 1.216$} & \multicolumn{2}{|l|}{$3.824 \pm 1.190$} & \multicolumn{2}{|l|}{0.668} & 0.664 \\
\hline TG (mmol/dl) & \multicolumn{2}{|l|}{$1.235 \pm 0.952$} & \multicolumn{2}{|l|}{$1.037 \pm 0.590$} & \multicolumn{2}{|l|}{0.384} & 0.384 \\
\hline Size (cm) & \multicolumn{2}{|l|}{$3.987 \pm 2.536$} & \multicolumn{2}{|l|}{$4.234 \pm 2.892$} & \multicolumn{2}{|l|}{0.533} & 0.534 \\
\hline AFP $>20 \mathrm{ng} / \mathrm{ml}$ & \multicolumn{2}{|l|}{$30(31.9 \%)$} & \multicolumn{2}{|l|}{$56(44.1 \%)$} & \multicolumn{2}{|l|}{$0.009^{\S}$} & $0.014{ }^{q}$ \\
\hline $\mathrm{HBsAg}+$ & \multicolumn{2}{|l|}{$71(75.5 \%)$} & \multicolumn{2}{|l|}{$89(70.1 \%)$} & \multicolumn{2}{|l|}{$0.355^{\S}$} & $0.228^{9}$ \\
\hline Stage $(0-B)^{\&}$ & \multicolumn{2}{|l|}{$72(76.6 \%)$} & \multicolumn{2}{|l|}{87 (68.5\%) } & \multicolumn{2}{|l|}{$0.0 .190^{\S}$} & $0.146^{\pi}$ \\
\hline rs13306731 & \multicolumn{2}{|l|}{$\begin{array}{l}\text { AA } \\
(n=107)\end{array}$} & \multicolumn{2}{|l|}{$\begin{array}{l}\text { AG\&GG } \\
(n=114)\end{array}$} & & & \\
\hline LDL (mmol/dl) & $2.262 \pm 0.846$ & & $2.376 \pm 0.943$ & & 0.993 & & 0.993 \\
\hline $\mathrm{HDL}(\mathrm{mmol} / \mathrm{dl})$ & $0.937 \pm 0.382$ & & $0.995 \pm 0.328$ & & 0.230 & & 0.219 \\
\hline TC (mmol/dl) & $3.749 \pm 1.176$ & & $3.880 \pm 1.221$ & & 0.921 & & 0.920 \\
\hline TG (mmol/dl) & $1.201 \pm 0.913$ & & $1.046 \pm 0.597$ & & 0.246 & & 0.246 \\
\hline size $(\mathrm{cm})$ & $3.999 \pm 2.514$ & & $4.253 \pm 2.951$ & & 0.518 & & 0.520 \\
\hline AFP $>20 \mathrm{ng} / \mathrm{ml}$ & $34(31.8 \%)$ & & $52(45.6 \%)$ & & $0.003^{\S}$ & & $0.010^{9}$ \\
\hline $\mathrm{HBsAg}+$ & $79(73.8 \%)$ & & $81(71.1 \%)$ & & $0.498^{\S}$ & & $0.352^{\pi}$ \\
\hline Stage $(0-B)^{\&}$ & $80(74.8 \%)$ & & 79 (69.3\%) & & $0.354^{\S}$ & & $0.311^{\natural}$ \\
\hline Haplotype & $\begin{array}{l}\text { CCA } \\
(n=285)\end{array}$ & $\begin{array}{l}\text { TGG } \\
(n=133)\end{array}$ & $\begin{array}{l}\text { TGA } \\
(n=24)\end{array}$ & TGG $P$ value ${ }^{\dagger}$ & TGG $P$ value ${ }^{\ddagger}$ & TGA $P$ value $e^{\ddagger}$ & TGA $P$ value ${ }^{\ddagger}$ \\
\hline LDL (mmol/dl) & $2.311 \pm 0.911$ & $2.349 \pm 0.905$ & $2.259 \pm 0.640$ & 0.702 & 0.774 & 0.785 & 0.863 \\
\hline $\mathrm{HDL}(\mathrm{mmol} / \mathrm{dl})$ & $0.955 \pm 0.375$ & $1.003 \pm 0.318$ & $0.912 \pm 0.300$ & 0.206 & 0.391 & 0.597 & 0.592 \\
\hline TC (mmol/dl) & $3.829 \pm 1.215$ & $3.862 \pm 1.221$ & $3.393 \pm 0.688$ & 0.802 & 0.927 & 0.091 & 0.100 \\
\hline TG (mmol/dl) & $1.178 \pm 0.854$ & $1.046 \pm 0.587$ & $0.870 \pm 0.467$ & 0.115 & 0.149 & 0.089 & 0.067 \\
\hline size (cm) & $4.131 \pm 2.715$ & $4.228 \pm 2.886$ & $3.500 \pm 2.077$ & 0.755 & 0.808 & 0.311 & 0.182 \\
\hline AFP $>20 \mathrm{ng} / \mathrm{ml}$ & $60(21.1 \%)$ & $38(28.6 \%)$ & $4(16.7 \%)$ & $0.042^{\S}$ & $0.065^{\pi}$ & $0.487^{\S}$ & $0.555^{9}$ \\
\hline $\mathrm{HBsAg}+$ & $206(72.3 \%)$ & 95 (71.4\%) & 17 (70.8\%) & $0.857^{\S}$ & $0.908^{\natural}$ & $0.879^{\S}$ & $0.634^{\natural}$ \\
\hline Stage $(0-B)^{\&}$ & 202 (70.9\%) & 92 (69.2\%) & 7 (29.2\%) & $0.562^{\S}$ & $0.593^{\natural}$ & $0.642^{\S}$ & $0.409^{n}$ \\
\hline
\end{tabular}

${ }^{\dagger}$ One-way ANOVA

${ }^{\ddagger}$ ANOVA adjusted for age and sex

SLogistic regression

"Logistic regression adjusted for age and sex

${ }^{\&}$ Stage $0-B$ versus C-D 


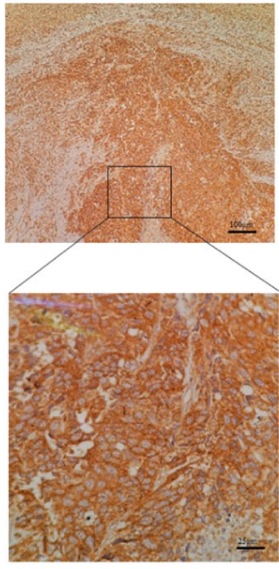

(A)

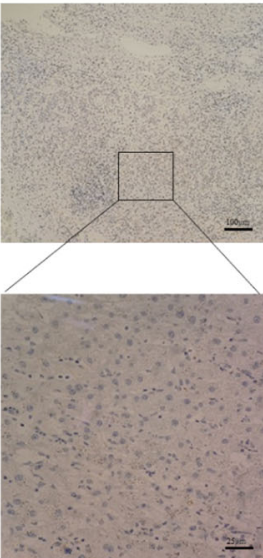

(B)

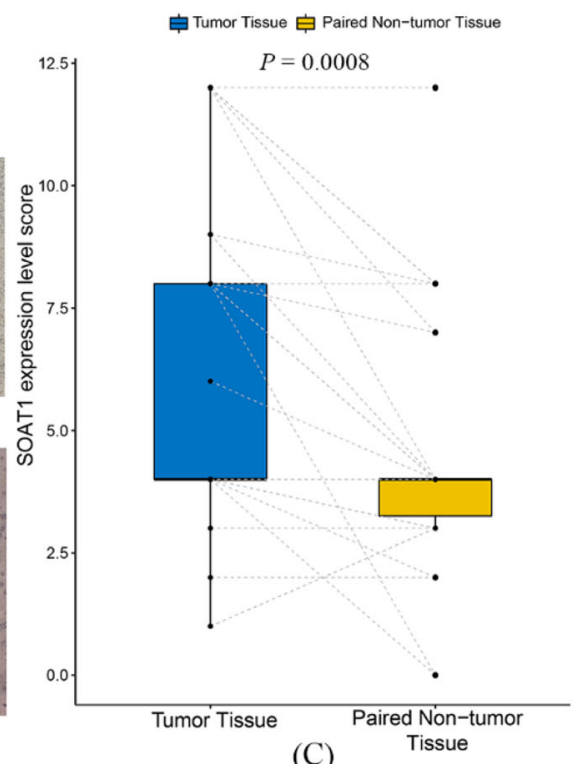

Fig. 2 SOAT1 protein expression in HCC tumor and non-tumor tissues. a Presentative tumor immunohistochemistry staining of SOAT1 protein; b Presentative non-tumor immunohistochemistry staining of SOAT1 protein; c IHC score differences between tumor and nontumor tissues $(P<0.001$, paired Wilcoxon singed-rank test). Tumor and non-tumor Samples from the same patient are connected with dotted gray lines. The thickness of the dotted line represents different sample sizes

Table 6 Association of SOAT1 expression with the characteristic of HCC patients

\begin{tabular}{|c|c|c|c|c|c|}
\hline & Low expression & High expression & $P$ value $^{\dagger}$ & OR & $\mathrm{Cl}(95 \%)$ \\
\hline \multicolumn{6}{|l|}{ rs10753191 } \\
\hline CC & 8 & 9 & Ref. & 1 & \\
\hline $\mathrm{CT}$ & 8 & 9 & 1 & 1 & $0.260-3.845$ \\
\hline$\pi$ & 3 & 5 & 1 & 1.481 & $0.265-8.267$ \\
\hline Dominant model & & & 1 & 1.131 & $0.328-3.898$ \\
\hline Recessive model & & & 0.709 & 0.675 & $0.139-3.283$ \\
\hline \multicolumn{6}{|l|}{ rs13306731 } \\
\hline AA & 9 & 9 & Ref. & 1 & \\
\hline$A G$ & 7 & 11 & 1 & 1 & $0.158-6346$ \\
\hline GG & 3 & 3 & 0.738 & 1.571 & $0.418-5.903$ \\
\hline Dominant model & & & 0.756 & 0.714 & $0.209-2.443$ \\
\hline Recessive model & & & 1 & 0.800 & $0.142-4.513$ \\
\hline \multicolumn{6}{|l|}{ Haplotype } \\
\hline CCA & $24(63.16 \%)$ & $27(58.70 \%)$ & Ref. & / & \\
\hline TGA & $1(2.63 \%)$ & $2(4.35 \%)$ & 1 & 1.778 & $0.151-20.863$ \\
\hline TGG & $13(34.21 \%)$ & 17 (36.96\%) & 0.819 & 1.162 & $0.469-2.881$ \\
\hline \multicolumn{6}{|l|}{ Stage } \\
\hline $0-B$ & 12 & 15 & Ref. & / & \\
\hline$C-D$ & 7 & 8 & 1 & 1.094 & $0.308-3.883$ \\
\hline
\end{tabular}


markedly higher expression level in tumor tissues compared to paired non-tumor tissues $(P<0.001$, paired Wilcoxon singed-rank test, Fig. 2). We found no significant association of the SOAT1 protein expression by genotype, haplotype or pathological stages (Table 6).

\section{Functionality of the SOAT1 SNPs}

SNPs rs10753191 (V323V) and rs3753526 (L475L) do not change amino acid, and Q526R (rs13306731) is a predicted benign amino acid change based on Polyphen2 program, (http://genetics.bwh.harvard.edu/pph2/index. shtml). We used the Position Weight Matrix model (PMW) based SNP2TFBS (https://ccg.epfl.ch/snp2tfbs/) in-silico bioinformatic tool to assess SNP impact on transcription factor binding (TFB) [24, 25]. While rs3753526 and rs13306731 have no effect on TFB, rs10753191 was predicted to significantly enhance binding to DNA-binding protein regulatory factor X-5 (RFX5). RFX5 has been reported to significantly upregulated in HCC tumors and cell lines [26]. Thus, rs10753191 may affect TFB and possibly gene regulation.

\section{HCC data from TCGA}

A high level of SOAT1 mRNA expression level was associated with a marginal significantly shorter overall survival in Asians $(P=0.046)$. However, there was not association in White/Caucasians $(P=0.58)$, or in all ethnic groups combined (Asian, White and black, $P=0.17$, Fig. 3). There was no significant impact of SOAT1 expression on OS when restricting the survival analysis to those HBV-infected $(n=150, P=0.32)$.

\section{Discussion}

In this study we found a protective association between two variant SOAT1 alleles and a haplotype carrying these alleles and HCC after adjusting for lipid levels. We also observed a markedly higher protein expression level of SOATlin tumor tissues compared to paired non-tumor tissues. A high SOAT1 mRNA expression level was further revealed to be associated with a shorter overall survival of HCC patients from the TCGA data in Asians but not in Caucasians, suggesting a population-specific role of SOAT1 in HCC. The ethnic/population specific role of SOAT1 is worthy particular attention as it is considered as a promising drug target of HCC [11]. SOAT1 is the key protein in catalyzing the formation of fatty acid-cholesterol esters [5] and we observed lower lipid levels in HCC cases compared to healthy controls. Previous studies suggest that cholesterol metabolism plays an important role in the progression of HCC [27-31]. A proteomics study found that HCC patients with disrupted cholesterol metabolism and high expression of SOAT1 tend to have a poorer prognosis [11]. The same study found that in a patient-derived tumor xenograft mouse model suppression of SOAT1 reduced tumor size [11]. Thus, SOAT1 may be a new target for HCC treatment. Our results suggest that SOAT1 may influence HCC risk through regulation of lipid metabolism.

We observed that LDL, HDL, TC and TG levels were lower in HCC cases than in normal controls. This finding is agreement with other studies which show that lipids and triglyceride levels are decreased in patients with HCC [27]. The relationship between lipid and HCC is complex. On the one hand, lipid metabolism alteration can be a consequence of HCC development. Cancer cachexia is frequently observed in cancer patients and characterizes by reduction in fat stores, elevated carbohydrate utilization and protein degradation. High growth rate of cancer cells leads to hypoxia and increased energy demand, and eventually promotes fatty-acid oxidation which will deplete fat storage [32-34]. On the other
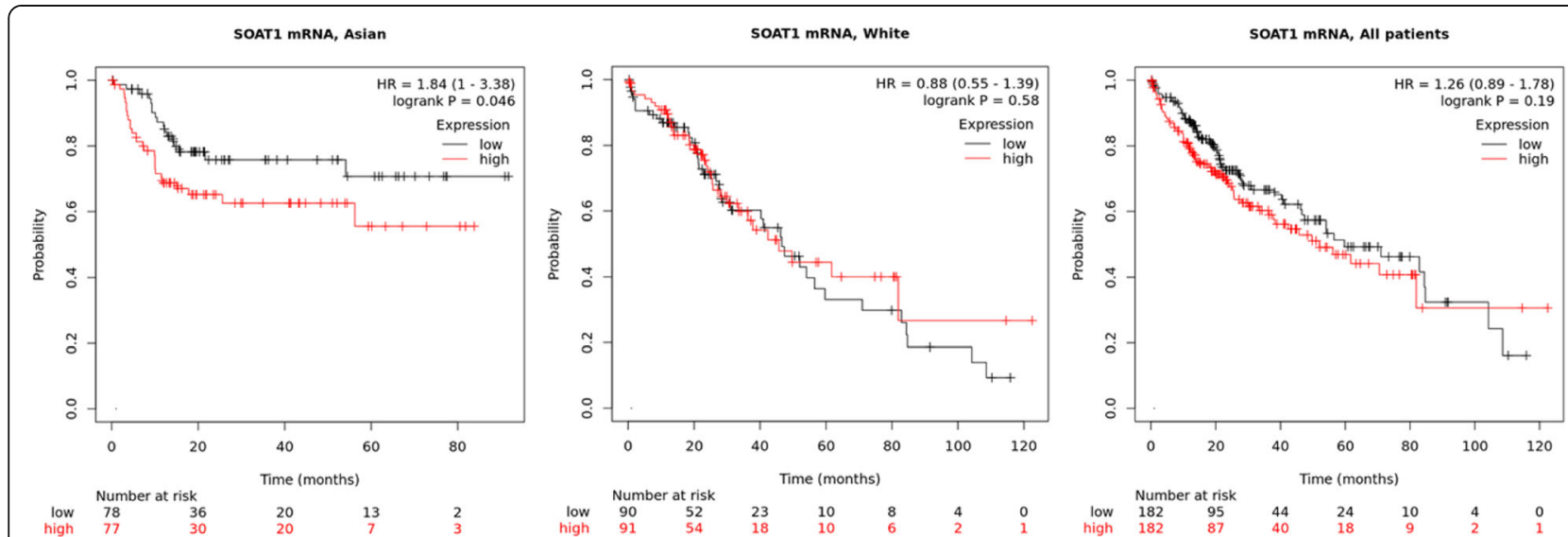

Fig. 3 Impact of SOAT1 mRNA expression level on survival of HCC patients in TCGA, stratified by ethnic group. a. Asian; b. White; c. In all patients. $H R$, hazard rate 
hand, dysregulated lipid metabolism may promote $\mathrm{HCC}$, due to impaired insulin and IGF-1 pro-tumorigenic growth factors [35, 36].

Several SOAT1 SNPs are associated with cholesterol metabolism [14, 15]. A meta-analysis by Andrew et al. found rs4421551 is associated with HDL level [14]. Wu et al. reported that carriers of rs1044925 variant genotypes had lower serum TC, LDL and ApoB levels than the reference genotype [15]. Both of these SNPs are in linkage disequilibrium with the three SNPs in this study $\left(D^{\prime}=1\right)$. It remains possible that the variant allele at rs10753191 or its proxy rs3753526 or other variants in LD with these SNPs may reduce the risk of HCC through lowering the lipid levels.

In an analysis of association between genotype and HCC related phenotypes, a lower level of AFP in serum decrease tended to associate with the SNPs employed in this study. Due to the low sensitivity and specificity of AFP for HCC diagnosis, this association may have utility in risk assessment.

Jiang et al. reported that a high SOAT1 expression increases the severity of HCC patients [11]. Our immunohistochemistry results also found higher expression of SOAT1 in tumor tissue compared to paired non-tumor tissue. However, our analysis showed no significant association between SOAT1 protein expression by genotypes, haplotypes or BCLC stages (Table 6).

Our results suggest SOAT1 variants may modestly modify HCC risk, possibly through the lipid metabolism pathway. On the other hand, our results also suggest that the impact of SOAT1 on HCC might be limited, calling for continuing search of other HCC host proteins involved in this multigenic heterogenous cancer.

Several limitations in this case-control study should be noted. HCC development is a complex process linked to multiple factors including age, sex, alcohol consumption, environment toxins, HBV and HCV viral levels, and diet. This study did not adjust for all of these confounding factors. Second, our sample size was not large enough to detect small effect sizes. Our sample size had adequate power of $80 \%$ to detect genotype relative risk of 1.88 for SNP rs10753191 (MAF 0.31, dominant model) or 2.05 for rs10753191(MAF 0.39) based on calculation using Genetic Association Study (GAS) Power Calculator [37]. Third, this study employed multiple genetic models and explanatory variables which could cause inflation of type 1 errors. The prior biological evidence of the gene-disease relationship is in favor of presence of a weak genetic association. Fourth, we only queried 2 independent SNPs and three haplotypes so it is quite likely that our SNPs and haplotypes are tracking through $\mathrm{LD}$ with other variants that may be functional. These SNPs may not be causal or functional by themselves. In addition, the frequencies of variants and haplotype structure of SOAT1 vary among populations, thus their effects in other populations may also vary. Finally, we did not have a replication cohort to validate our results; therefore, further studies are warranted to validate our results and to identify putative causal variants through fine mapping and functional studies. Potential relationship of HBV infection interacting with SOAT1 to contribute to HCC tumorigenesis is also an important topic for the future research.

\section{Conclusion}

In conclusion, this study is the first to implicate SOAT1 genetic variation that modifies HCC susceptibility. Studies with larger sample size, stratified by confounding factors and protein levels of SOAT1, should be conducted to validate its role in developing of HCC.

\section{Abbreviations \\ HBV: Hepatitis B virus; HCC: Hepatocellular carcinoma; SOAT: Sterol O- acyltransferase; SNP: Single Nucleotide Polymorphism; NAFLD: Non-alcoholic fatty liver disease; FFPE: Formalin-fixed and paraffin-embedded; \\ BCLC: Barcelona Clinic Liver Cancer; MAF: Minor allele frequency; LD: Linkage disequilibrium; LDL: Low density lipoprotein; HDL: High density lipoprotein; TC: Total cholesterol; TG: Triglyceride; AFP: Alpha-fetoprotein; \\ HBsAg: Hepatitis B s antigen}

\section{Supplementary Information}

The online version contains supplementary material available at https://doi. org/10.1186/s12885-021-08245-1.

\section{Additional file 1}

\section{Acknowledgments}

We thank Dr. Peng and other doctors in the Department of Pathology, First Hospital of Wenzhou Medical University for their support in handling pathological specimens.

\section{Authors' contributions}

AP, CAW and LJX designed the study and prepared the manuscript. CYL assayed SNP genotypes, performed data analysis and prepared the first draft. YXJ collected samples and data, performed data quality control and data analysis. CY and CGR analyzed and interpreted the pathological and $\mathrm{IHC}$ data. All authors have read and approved the final version of the manuscript.

\section{Funding}

This project has been funded in whole or in part with Federal funds from the Frederick National Laboratory for Cancer Research, National Institutes of Health, under contract HHSN261200800001E. This research was supported in part by the Intramural Research Program of NIH, Frederick National Lab, Center for Cancer Research. The content of this publication does not necessarily reflect the views or policies of the Department of Health and Human Services, nor does mention of trade names, commercial products or organizations imply endorsement by the US Government. The funding bodies had no role in the design of the study and collection, analysis, and interpretation of data and in writing the manuscript.

\section{Availability of data and materials}

The datasets used and/or analyzed during the current study are available from the corresponding author on reasonable request.

\section{Declarations}

\section{Ethics approval and consent to participate}

The study was conducted in accordance with the Declaration of Helsinki. The Ethics Committee of Wenzhou Medical University approved this study and the study protocols. Written informed consents were obtained from individuals of healthy controls. An IRB exemption was obtained from the National Institutes of 
Health Office of Human Subjects Research (OHSRP Review \#12836) for using archived pathological specimens and the de-identified health information.

\section{Consent for publication}

Not applicable.

\section{Competing interests}

The authors declare that they have no competing interests.

\section{Author details}

${ }^{1}$ Key Laboratory of Laboratory Medicine, Ministry of Education, Zhejiang Provincial Key Laboratory of Medical Genetics, Wenzhou Medical University, Wenzhou, Zhejiang, China. 'Department of Laboratory Medicine, The Second Affiliated Hospital \& Yuying Children's Hospital of Wenzhou Medical University, Wenzhou, Zhejiang, China. ${ }^{3}$ Department of Pathology, The First Affiliated Hospital of Wenzhou Medical University, Wenzhou, Zhejiang, China. ${ }^{4}$ Basic Science Program, Frederick National Laboratory for Cancer Research, Frederick, MD, USA. ${ }^{5}$ Zhejiang Provincial People's Hospital, Affiliated Hospital of Hangzhou Medical College, Zhejiang, Hangzhou, China.

Received: 21 September 2020 Accepted: 23 April 2021

Published online: 26 May 2021

\section{References}

1. Bray F, Ferlay J, Soerjomataram I, Siegel RL, Torre LA, Jemal A. Global cancer statistics 2018: GLOBOCAN estimates of incidence and mortality worldwide for 36 cancers in 185 countries. CA Cancer J Clin. 2018;68(6):394-424. https://doi.org/10.3322/caac.21492.

2. McGlynn KA, Petrick JL, London WT. Global epidemiology of hepatocellular carcinoma: an emphasis on demographic and regional variability. Clin Liver Dis. 2015;19(2):223-38. https://doi.org/10.1016/j.cld.2015.01.001.

3. Younossi ZM, Otgonsuren M, Henry L, Venkatesan C, Mishra A, Erario $M$, et al. Association of nonalcoholic fatty liver disease (NAFLD) with hepatocellular carcinoma (HCC) in the United States from 2004 to 2009. Hepatology. 2015;62(6):1723-30. https://doi.org/10.1002/hep.2 8123

4. Beste LA, Leipertz SL, Green PK, Dominitz JA, Ross D, loannou GN. Trends in Burden of Cirrhosis and Hepatocellular Carcinoma by Underlying Liver Disease in US Veterans, 2001-2013. Gastroenterology. 2015;149(6):1471-1482.e1475.

5. Chang T, Chang CC, Lin S, Yu C, Li BL, Miyazaki A. Roles of acyl-coenzyme a: cholesterol acyltransferase-1 and -2. Curr Opin Lipidol. 2001;12(3):289-96. https://doi.org/10.1097/00041433-200106000-00008.

6. Ioannou GN. The role of cholesterol in the pathogenesis of NASH. Trends Endocrinol Metab. 2016;27(2):84-95. https://doi.org/10.1016/j. tem.2015.11.008.

7. Katsuren K, Tamura T, Arashiro R, Takata K, Matsuura T, Niikawa N, et al. Structure of the human acyl-CoA:cholesterol acyltransferase-2 (ACAT-2) gene and its relation to dyslipidemia. Biochim Biophys Acta. 2001;1531(3): 230-40. https://doi.org/10.1016/S1388-1981(01)00106-8.

8. Kushwaha RS, Rosillo A, Rodriguez R, McGill HC. Expression levels of ACAT1 and ACAT2 genes in the liver and intestine of baboons with high and low lipemic responses to dietary lipids. J Nutr Biochem. 2005;16(12):714-21. https://doi.org/10.1016/j.jnutbio.2005.03.010.

9. Chang C, Sakashita N, Ornvold K, Lee O, Chang E, Dong R, et al. Immunological quantitation and localization of ACAT-1 and ACAT-2 in human liver and small intestine. J Biol Chem. 2000;275(36):28083-92. https://doi.org/10.1074/jbc.M003927200.

10. Joyce CW, Shelness GS, Davis MA, Lee RG, Skinner K, Anderson RA, et al. ACAT1 and ACAT2 membrane topology segregates a serine residue essential for activity to opposite sides of the endoplasmic reticulum membrane. Mol Biol Cell. 2000;11(11):3675-87. https://doi.org/10.1091/ mbc.11.11.3675.

11. Jiang $Y$, Sun A, Zhao $Y$, Ying W, Sun $H$, Yang $X$, et al. Proteomics identifies new therapeutic targets of early-stage hepatocellular carcinoma. Nature. 2019;567(7747):257-61. https://doi.org/10.1038/s41586-019-0987-8.

12. Chan L-K, Ng IO-L. Proteomic profiling in liver cancer: another new page. Transl Gastroenterol Hepatol. 2019;4:47. https://doi.org/10.21037/ tgh.2019.06.03.
13. Khatib SA, Wang XW. Proteomic heterogeneity reveals SOAT1 as a potential biomarker for hepatocellular carcinoma. Transl Gastroenterol Hepatol. 2019; 4:37. https://doi.org/10.21037/tgh.2019.05.09.

14. Edmondson AC, Braund PS, Stylianou IM, Khera AV, Nelson CP, Wolfe ML, et al. Dense genotyping of candidate gene loci identifies variants associated with high-density lipoprotein cholesterol. Circ Cardiovasc Genet. 2011;4(2): 145-55. https://doi.org/10.1161/CIRCGENETICS.110.957563.

15. Wu D-F, Yin R-X, LHH A, Hu X-J, Cao X-L, Miao L, et al. Polymorphism of rs1044925 in the acyl-CoA:cholesterol acyltransferase-1 gene and serum lipid levels in the Guangxi Bai Ku Yao and Han populations. Lipids Health Dis. 2010;9(1):139. https://doi.org/10.1186/1476-511X-9-139.

16. Yang $X$, Guo X, Chen Y, Chen G, Ma Y, Huang K, et al. Telomerase reverse transcriptase promoter mutations in hepatitis $B$ virus-associated hepatocellular carcinoma. Oncotarget. 2016;7(19):27838-47. https://doi.org/1 0.18632/oncotarget.8539.

17. Pikor LA, Enfield KSS, Cameron H, Lam WL. DNA extraction from paraffin embedded material for genetic and epigenetic analyses. J Vis Exp. 2011;49.

18. Kinoshita A, Onoda H, Fushiya N, Koike K, Nishino H, Tajiri H. Staging systems for hepatocellular carcinoma: current status and future perspectives. World J Hepatol. 2015;7(3):406-24. https://doi.org/10.4254/wjh.v7.i3.406.

19. Consortium GP, Auton A, Brooks LD, Durbin RM, Garrison EP, Kang HM, et al. A global reference for human genetic variation. Nature. 2015;526(7571):6874. https://doi.org/10.1038/nature15393.

20. Team RDC. R: a language and environment for statistical computing: reference index. Vienna: R Foundation for Statistical Computing; 2010.

21. Shin J-H, Blay S, McNeney B, Graham J. LDheatmap: An R function for graphical display of pairwise linkage disequilibria between single nucleotide polymorphisms. J Stat Softw. 2006;16(1):1-9.

22. Sinnwell JP, Schaid DJ. Haplo.stats: Statistical analysis of haplotypes with traits and covariates when linkage phase is ambiguous. $\mathrm{R}$ packag, version 1. 8.6. https://cran.r-project.org/package=haplo.stats.

23. Menyhart O, Nagy A, Gyorffy B. Determining consistent prognostic biomarkers of overall survival and vascular invasion in hepatocellular carcinoma. R Soc Open Sci. 2018;5(12):181006. https://doi.org/10.1098/rsos.181006.

24. Mathelier A, Zhao X, Zhang AW, Parcy F, Worsley-Hunt R, Arenillas DJ, et al. JASPAR 2014: an extensively expanded and updated open-access database of transcription factor binding profiles. Nucleic Acids Res. 2014;42(Database issue):D142-7. https://doi.org/10.1093/nar/gkt997.

25. Kumar S, Ambrosini G, Bucher P. SNP2TFBS - a database of regulatory SNPs affecting predicted transcription factor binding site affinity. Nucleic Acids Res. 2017:45(D1):D139-44. https://doi.org/10.1093/nar/gkw1064.

26. Zhao $Y$, Xie X, Liao W, Zhang H, Cao H, Fei R, et al. The transcription factor RFX5 is a transcriptional activator of the TPP1 gene in hepatocellular carcinoma. Oncol Rep. 2017;37(1):289-96. https://doi.org/1 0.3892/or.2016.5240.

27. Jiang J, Nilsson-Ehle P, Xu N. Influence of liver cancer on lipid and lipoprotein metabolism. Lipids Health Dis. 2006;5(1):4. https://doi.org/10.11 86/1476-511X-5-4

28. Lu M, Hu X-H, Li Q, Xiong Y, Hu G-J, Xu J-J, et al. A specific cholesterol metabolic pathway is established in a subset of HCCs for tumor growth. J Mol Cell Biol. 2013;5(6):404-15. https://doi.org/10.1093/jmcb/mjt039.

29. Jiang S-S, Weng D-S, Jiang L, Zhang Y-J, Pan K, Pan Q-Z, et al. The clinical significance of preoperative serum cholesterol and high-density lipoproteincholesterol levels in hepatocellular carcinoma. J Cancer. 2016;7(6):626-32. https://doi.org/10.7150/jca.13837.

30. Carr Bl, Giannelli G, Guerra V, Giannini EG, Farinati F, Rapaccini GL, et al. Plasma cholesterol and lipoprotein levels in relation to tumor aggressiveness and survival in HCC patients. Int J Biol Markers. 2018;33(4): 423-31. https://doi.org/10.1177/1724600818776838.

31. Teng C-F, Hsieh W-C, Yang C-W, Su H-M, Tsai T-F, Sung W-C, et al. A biphasic response pattern of lipid metabolomics in the stage progression of hepatitis B virus X tumorigenesis. Mol Carcinog. 2016;55(1):105-14. https:// doi.org/10.1002/mc.22266.

32. Ackerman D, Simon MC. Hypoxia, lipids, and cancer: surviving the harsh tumor microenvironment. Trends Cell Biol. 2014;24(8):472-8. https://doi. org/10.1016/j.tcb.2014.06.001.

33. Santos CR, Schulze A. Lipid metabolism in cancer. FEBS J. 2012;279(15): 2610-23. https://doi.org/10.1111/j.1742-4658.2012.08644.x.

34. Shaw JH, Wolfe RR. Fatty acid and glycerol kinetics in septic patients and in patients with gastrointestinal cancer. The response to glucose infusion and 
parenteral feeding. Ann Surg. 1987;205(4):368-76. https://doi.org/10.1097/ 00000658-198704000-00005.

35. Samuel VT, Shulman GI. Mechanisms for insulin resistance: common threads and missing links. Cell. 2012;148(5):852-71. https://doi.org/10.1016/j.cell.2012. 02.017 .

36. Renehan AG, Frystyk J, Flyvbjerg A. Obesity and cancer risk: the role of the insulin-IGF axis. Trends Endocrinol Metab. 2006;17(8):328-36. https://doi. org/10.1016/.jtem.2006.08.006.

37. Skol AD, Scott $L$, Abecasis GR, Boehnke M. Joint analysis is more efficient than replication-based analysis for two-stage genome-wide association studies. Nat Genet. 2006;38(2):209-13. https://doi.org/10.1038/ng1706.

\section{Publisher's Note}

Springer Nature remains neutral with regard to jurisdictional claims in published maps and institutional affiliations.

Ready to submit your research? Choose BMC and benefit from:

- fast, convenient online submission

- thorough peer review by experienced researchers in your field

- rapid publication on acceptance

- support for research data, including large and complex data types

- gold Open Access which fosters wider collaboration and increased citations

- maximum visibility for your research: over $100 \mathrm{M}$ website views per year

At $\mathrm{BMC}$, research is always in progress.

Learn more biomedcentral.com/submissions 